\title{
Joint Discrete Approximation of a Pair of Analytic Functions by Periodic Zeta-Functions
}

\author{
Aidas Balčiūnas ${ }^{a}$, Virginija Garbaliauskienè ${ }^{b}$, \\ Julija Karaliūnaite $\dot{e}^{c}$, Renata Macaitienè $\dot{e}^{b}$, \\ Jurgita Petuškinaite் $\dot{\mathrm{e}}^{a}$ and Audronè Rimkevičiené $\dot{\mathrm{e}}^{d}$ \\ ${ }^{a}$ Faculty of Mathematics and Informatics, Vilnius University \\ Naugarduko str. 24, LT-03225 Vilnius, Lithuania \\ ${ }^{b}$ Institute of Regional Development, Šiauliai University \\ P. Višinskio str. 25, LT-76351 Šiauliai, Lithuania \\ ${ }^{c}$ Faculty of Fundamental Sciences, Vilnius Gediminas Technical University \\ Saulètekio av. 11, LT-10223 Vilnius, Lithuania \\ ${ }^{d}$ Faculty of Business and Technologies, Šiauliai State College \\ Aušros av. 40, LT-76241 Šiauliai, Lithuania \\ E-mail: aidas.balciunas@mif.vu.lt \\ E-mail(corresp.): virginija.garbaliauskiene@su.lt \\ E-mail: julija.karaliunaite@vgtu.lt \\ E-mail: renata.macaitiene@su.lt \\ E-mail: j.petuskinaite@gmail.com \\ E-mail: a.rimkeviciene@svako.lt
}

Received June 7, 2019; revised November 1, 2019; accepted November 1, 2019

\begin{abstract}
In the paper, the problem of simultaneous approximation of a pair of analytic functions by a pair of discrete shifts of the periodic and periodic Hurwitz zeta-function is considered. The above shifts are defined by using the sequence of imaginary parts of non-trivial zeros of the Riemann zeta-function. For the proof of approximation theorems, a weak form of the Montgomery pair correlation conjecture is applied.
\end{abstract}

Keywords: Hurwitz zeta-function, non-trivial zeros of the Riemann zeta-function, periodic zeta-function, periodic Hurwitz zeta-function, universality.

AMS Subject Classification: 11M06; 11M35; 11M41.

Copyright (c) 2020 The Author(s). Published by VGTU Press

This is an Open Access article distributed under the terms of the Creative Commons Attribution License (http://creativecommons.org/licenses/by/4.0/), which permits unrestricted use, distribution, and reproduction in any medium, provided the original author and source are credited. 


\section{Introduction}

In the paper, we consider the approximation of a pair of analytic functions by shifts of the periodic and periodic Hurwitz zeta-functions involving imaginary parts of non-trivial zeros of the Riemann zeta-function. We recall the definitions of the mentioned zeta-functions. Let $s=\sigma+i t$ be a complex variable, and $\mathfrak{a}=\left\{a_{m}: m \in \mathbb{N}\right\}$ and $\mathfrak{b}=\left\{b_{m}: m \in \mathbb{N}_{0}=\mathbb{N} \cup\{0\}\right\}$ be two periodic sequences of complex numbers with minimal periods $q_{1} \in \mathbb{N}$ and $q_{2} \in \mathbb{N}$, respectively. Then the periodic zeta-function $\zeta(s ; \mathfrak{a})$ and the periodic Hurwitz zeta-function $\zeta(s, \alpha ; \mathfrak{b})$ with parameter $\alpha, 0<\alpha \leq 1$, are defined, for $\sigma>1$, by the Dirichlet series

$$
\zeta(s ; \mathfrak{a})=\sum_{m=1}^{\infty} \frac{a_{m}}{m^{s}} \text { and } \zeta(s, \alpha ; \mathfrak{b})=\sum_{m=0}^{\infty} \frac{b_{m}}{(m+\alpha)^{s}}
$$

If $a_{m} \equiv 1$, then $\zeta(s ; \mathfrak{a})$ reduces to the Riemann zeta-function $\zeta(s)=\sum_{m=1}^{\infty} \frac{1}{m^{s}}$, $\sigma>1$, and $\zeta(s, \alpha ; \mathfrak{b})$, for $b_{m} \equiv 1$, becomes the classical Hurwitz zeta-function $\zeta(s ; \alpha)=\sum_{m=0}^{\infty} 1 /(m+\alpha)^{s}$. The periodicity of the sequences $\mathfrak{a}$ and $\mathfrak{b}$ implies the equalities

$$
\begin{aligned}
& \zeta(s ; \mathfrak{a})=\frac{1}{q_{1}^{s}} \sum_{m=1}^{q_{1}} a_{m} \zeta\left(s, \frac{m}{q_{1}}\right) \\
& \zeta(s, \alpha ; \mathfrak{b})=\frac{1}{q_{2}^{s}} \sum_{m=0}^{q_{2}-1} b_{m} \zeta\left(s, \frac{m+\alpha}{q_{2}}\right) .
\end{aligned}
$$

Thus, the well-known properties of the Hurwitz zeta-function show that the functions $\zeta(s ; \mathfrak{a})$ and $\zeta(s, \alpha ; \mathfrak{b})$ have analytic continuation to the whole complex plane, except for the point $s=1$ that is a simple pole with residues

$$
\frac{1}{q_{1}} \sum_{m=1}^{q_{1}} a_{m} \text { and } \frac{1}{q_{2}} \sum_{m=0}^{q_{2}-1} b_{m}
$$

respectively. If the above quantities are zero, then the corresponding zetafunctions are entire. The approximation of analytic functions by the functions $\zeta(s ; \mathfrak{a})$ and $\zeta(s, \alpha ; \mathfrak{b})$ was studied in $[8,26,28,29]$ and $[2,7,18,22,24,25,27]$, respectively.

The first joint results for a pair of functions $(\zeta(s ; \mathfrak{a}), \zeta(s, \alpha ; \mathfrak{b}))$ has been obtained in [9]. Assuming that the sequence $\mathfrak{a}$ is multiplicative, i. e., $a_{1}=1$ and $a_{m n}=a_{m} a_{n}$ for all coprimes $m$ and $n$, and that the parameter is transcendental, a joint universality theorem on the approximation of a pair of analytic functions has been proved. Let $D=\left\{s \in \mathbb{C}: \frac{1}{2}<\sigma<1\right\}, \mathcal{K}$ be the class of compact subsets of the strip $D$ with connected complements, $H(K)$ with $K \in \mathcal{K}$ be the class of continuous functions on $K$ that are analytic in the interior of $K$, and let $H_{0}(K)$ denote the subclass of $H(K)$ of non-vanishing functions. Then it was proved in [9] that if $K_{1}, K_{2} \in \mathcal{K}, f_{1}(s) \in H_{0}\left(K_{1}\right)$ and 
$f_{2}(s) \in H\left(K_{2}\right)$, then for every $\varepsilon>0$,

$$
\begin{gathered}
\liminf _{T \rightarrow \infty} \frac{1}{T} \operatorname{meas}\left\{\tau \in[0, T]: \sup _{s \in K_{1}}\left|\zeta(s+i \tau ; \mathfrak{a})-f_{1}(s)\right|<\varepsilon,\right. \\
\left.\sup _{s \in K_{2}}\left|\zeta(s+i \tau, \alpha ; \mathfrak{b})-f_{2}(s)\right|<\varepsilon\right\}>0
\end{gathered}
$$

where meas $A$ denotes the Lebesgue measure of a measurable set $A \subset \mathbb{R}$. A discrete version of the latter theorem has been presented in [15]. Let $\# A$ denote the cardinality of the set $A, N$ run over non-negative integers, and $\mathbb{P}$ be the set of all prime numbers. For $h>0$, define

$$
L(\mathbb{P}, \alpha, h, \pi)=\left\{(\log p: p \in \mathbb{P}),\left(\log (m+\alpha): m \in \mathbb{N}_{0}\right), \frac{2 \pi}{h}\right\} .
$$

If the set $L(\mathbb{P}, \alpha, h, \pi)$ is linearly independent over the field of rational numbers $\mathbb{Q}$, and the sequence $\mathfrak{a}$ is multiplicative, then, for the same $K_{1}, K_{2}$ and $f_{1}(s), f_{2}(s)$ as above, it was proved in [15] that, for every $\varepsilon>0$,

$$
\begin{gathered}
\liminf _{N \rightarrow \infty} \frac{1}{N+1} \#\left\{0 \leq k \leq N: \sup _{s \in K_{1}}\left|\zeta(s+i k h ; \mathfrak{a})-f_{1}(s)\right|<\varepsilon,\right. \\
\left.\sup _{s \in K_{2}}\left|\zeta(s+i k h, \alpha ; \mathfrak{b})-f_{2}(s)\right|<\varepsilon\right\}>0 .
\end{gathered}
$$

Moreover, under hypothesis that the set

$$
\left\{\left(h_{1} \log p: p \in \mathbb{P}\right),\left(h_{2} \log (m+\alpha): m \in \mathbb{N}_{0}, 2 \pi\right\}\right.
$$

is linearly independent over $\mathbb{Q}$, it was obtained the following modification of inequality (1.3):

$$
\begin{gathered}
\liminf _{N \rightarrow \infty} \frac{1}{N+1} \#\left\{0 \leq k \leq N: \sup _{s \in K_{1}}\left|\zeta\left(s+i k h_{1} ; \mathfrak{a}\right)-f_{1}(s)\right|<\varepsilon,\right. \\
\left.\sup _{s \in K_{2}}\left|\zeta\left(s+i k h_{2}, \alpha ; \mathfrak{b}\right)-f_{2}(s)\right|<\varepsilon\right\}>0 .
\end{gathered}
$$

Similar results also are given in [17] and [19]. Approximation results for more general collections consisting from periodic zeta functions were obtained in $[3,11,12,13,14,16,20]$ and $[23]$.

The aim of this paper is to replace in shifts $\zeta(s+i k h ; \mathfrak{a})$ and $\zeta(s+i k h ; \alpha ; \mathfrak{b})$ the sequence $\{k h\}$ by more complicated one. Let $0<\gamma_{1}<\gamma_{2}<\ldots \leq \gamma_{k} \leq \ldots$ be the sequence of imaginary parts of non-trivial zeros of the Riemann zetafunction. The behaviour of the sequence $\left\{\gamma_{k}: k \in \mathbb{N}\right\}$ is mysterious, therefore, we will use a certain hypothesis that is implied by the well-known Montgomery pair correlation conjecture [33]. Namely, we suppose that the estimate

$$
\sum_{\substack{\gamma_{k} \leq T \\\left|\gamma_{k}-\gamma_{l}\right|<\frac{c}{\log T}}} \sum_{\substack{\gamma_{l} \leq T \\ \log }} 1 \ll T \log T
$$


holds for $c>0$ as $T \rightarrow \infty$. The Montgomery conjecture gives the asymptotic formula for the left-hand side of (1.4). The condition (1.4) was applied in [6] for the approximation of analytic functions by shifts $\zeta\left(s+i \gamma_{k} h\right)$, in [30] for shifts $\zeta\left(s+i \gamma_{n} h, \alpha\right)$ and by shifts $\left.\left(\zeta\left(s+i \gamma_{k} h\right), \zeta\left(s+i \gamma_{k} h, \alpha\right)\right)\right)$ in [21]. In [4,5], in place of (1.4), the Riemann hypothesis was used. The paper [26] is devoted to joint approximation of analytic functions by shifts of Dirichlet $L$-functions $L\left(s+i \gamma_{k} h, \chi_{1}\right), \ldots, L\left(s+i \gamma_{k} h, \chi_{r}\right)$ also by using (1.4).

Now, we state the main theorems of the paper.

Theorem 1. Suppose that the sequence $\mathfrak{a}$ is multiplicative, the parameter $\alpha$ is transcendental, and the bound (1.4) is true. Let $K_{1}, K_{2} \in \mathcal{K}, f_{1}(s) \in H_{0}\left(K_{1}\right)$, $f_{2}(s) \in H\left(K_{2}\right)$ and $h>0$. Then, for every $\varepsilon>0$,

$$
\begin{gathered}
\liminf _{N \rightarrow \infty} \frac{1}{N} \#\left\{1 \leq k \leq N: \sup _{s \in K_{1}}\left|\zeta\left(s+i \gamma_{k} h ; \mathfrak{a}\right)-f_{1}(s)\right|<\varepsilon,\right. \\
\left.\sup _{s \in K_{2}}\left|\zeta\left(s+i \gamma_{k} h, \alpha ; \mathfrak{b}\right)-f_{2}(s)\right|<\varepsilon\right\}>0 .
\end{gathered}
$$

The positivity of a lower density of the set of shifts approximating a given pair $\left(f_{1}(s), f_{2}(s)\right)$ can be replaced by that of the density with some exception for $\varepsilon>0$. More precisely, the following statement is true.

Theorem 2. Under hypotheses of Theorem 1, the limit

$$
\begin{gathered}
\lim _{N \rightarrow \infty} \frac{1}{N} \#\left\{1 \leq k \leq N: \sup _{s \in K_{1}}\left|\zeta\left(s+i \gamma_{k} h ; \mathfrak{a}\right)-f_{1}(s)\right|<\varepsilon,\right. \\
\left.\sup _{s \in K_{2}}\left|\zeta\left(s+i \gamma_{k} h, \alpha ; \mathfrak{b}\right)-f_{2}(s)\right|<\varepsilon\right\}>0
\end{gathered}
$$

exists for all but at most countably many $\varepsilon>0$.

For the proof of Theorems 1 and 2, the Fourier transform and weak convergence methods will be applied.

\section{Uniform distribution modulo 1}

In this section, we present some facts related to the uniform distribution modulo 1 of sequences of real numbers.

We recall that the sequence $\left\{x_{k}: k \in \mathbb{N}\right\} \subset \mathbb{R}$ is uniformly distributed modulo 1 if, for every interval $[a, b) \subset[0,1)$

$$
\lim _{n \rightarrow \infty} \frac{1}{n} \sum_{k=1}^{n} \chi_{[a, b)}\left(\left\{x_{k}\right\}\right)=b-a,
$$

where $\chi_{[a, b)}$ is the indicator function of the interval $[a, b)$, and $\left\{x_{k}\right\}$ denotes the fractional part of $x_{k}$.

The next lemma is the well-known Weyl criterion. 
Lemma 1. A sequence $\left\{x_{k}: k \in \mathbb{N}\right\} \subset \mathbb{R}$ is uniformly distributed modulo 1 if and only if, for every $m \in \mathbb{Z} \backslash\{0\}$,

$$
\lim _{n \rightarrow \infty} \frac{1}{n} \sum_{k=1}^{n} e^{2 \pi i m x_{k}}=0 .
$$

Proof of the lemma can be found, for example, in [10].

Lemma 2. The sequence $\left\{\gamma_{k} a: k \in \mathbb{N}\right\}$ with every $a \in \mathbb{R} \backslash\{0\}$ is uniformly distributed modulo 1.

Proof. The lemma was obtained in [34] and used in [6].

Lemmas 1 and 2 will be applied for weak convergence of probability measures on certain topological groups. Let $\gamma=\{s \in \mathbb{C}:|s|=1\}$, and

$$
\Omega_{1}=\prod_{p \in \mathbb{P}} \gamma_{p} \text { and } \Omega_{2}=\prod_{m \in \mathbb{N}_{0}} \gamma_{m}
$$

where $\gamma_{p}=\gamma$ for all $p \in \mathbb{P}$ and $\gamma_{m}=\gamma$ for all $m \in \mathbb{N}_{0}$. In view of the Tikhonov theorem, $\Omega_{1}$ and $\Omega_{2}$, with the product topology and pointwise multiplication, are compact topological Abelian groups. Define $\Omega=\Omega_{1} \times \Omega_{2}$. Then again, $\Omega$ is a compact topological group, therefore, on $(\Omega, \mathcal{B}(\Omega))(\mathcal{B}(\mathbb{X})$ is the Borel $\sigma$-field of the space $\mathbb{X})$ the probability Haar measure $m_{H}$ exists, and we have the probability space $\left(\Omega, \mathcal{B}(\Omega), m_{H}\right)$. Denote by $\omega_{1}(p)$ the $p t h$ component of an element $\omega_{1} \in \Omega_{1}, p \in \mathbb{P}$, and by $\omega_{2}(m)$ the $m t h$ component of an element $\omega_{2} \in \Omega_{2}$. Elements of $\Omega$ are denoted by $\omega=\left(\omega_{1}, \omega_{2}\right), \omega_{1} \in \Omega_{1}, \omega_{2} \in \Omega_{2}$.

For $A \in \mathcal{B}(\Omega)$, define

$$
Q_{N, \alpha}(A)=\frac{1}{N} \#\left\{1 \leq k \leq N:\left(\left(p^{-i \gamma_{k} h}: p \in \mathbb{P}\right),(m+\alpha)^{-i \gamma_{k} h}: m \in \mathbb{N}_{0}\right) \in A\right\} \text {. }
$$

The next lemma deals with weak convergence of $Q_{N, \alpha}$ as $N \rightarrow \infty$.

Lemma 3. Suppose that $\alpha$ is a transcendental number. Then $Q_{N, \alpha}$ converges weakly to the Haar measure $m_{H}$ as $N \rightarrow \infty$.

Proof. We apply the Fourier transform method. Let $g_{N, \alpha}(\underline{k}, \underline{l}), \underline{k}=\left(k_{p}: k_{p} \in\right.$ $\mathbb{Z}, p \in \mathbb{P}), \underline{l}=\left(l_{m}: l_{m} \in \mathbb{Z}, m \in \mathbb{N}_{0}\right)$, be the Fourier transform of $Q_{N, \alpha}$. Then it is well known that

$$
g_{N, \alpha}(\underline{k}, \underline{l})=\int_{\Omega}\left(\prod_{p \in \mathbb{P}}^{\prime} \omega_{1}^{k_{p}}(p) \prod_{m \in \mathbb{N}_{0}}^{\prime} \omega_{2}^{l_{m}}(m)\right) \mathrm{d} Q_{N, \alpha},
$$

where "' means that only a finite number of integers $k_{p}$ and $l_{m}$ are distinct from zero. Thus, by the definition of $Q_{N, \alpha}$,

$$
\begin{aligned}
g_{N, \alpha}(\underline{k}, \underline{l}) & =\frac{1}{N} \sum_{k=1}^{N} \prod_{p \in \mathbb{P}}^{\prime} p^{-i h k_{p} \gamma_{k}} \prod_{m \in \mathbb{N}_{0}}^{\prime}(m+\alpha)^{-i h l_{m} \gamma_{k}} \\
& =\frac{1}{N} \sum_{k=1}^{N} \exp \left\{-i h \gamma_{k}\left(\sum_{p \in \mathbb{P}}^{\prime} k_{p} \log p+\sum_{m \in \mathbb{N}_{0}}^{\prime} l_{m} \log (m+\alpha)\right)\right\} .
\end{aligned}
$$


Clearly,

$$
g_{N, \alpha}(\underline{0}, \underline{0})=1 .
$$

Since $\alpha$ is transcendental, the set

$$
\left\{(\log p: p \in \mathbb{P}),\left(\log (m+\alpha): m \in \mathbb{N}_{0}\right)\right\}
$$

is linearly independent over $\mathbb{Q}[9]$. Therefore,

$$
\sum_{p \in \mathbb{P}}^{\prime} k_{p} \log p+\sum_{m \in \mathbb{N}_{0}}^{\prime} l_{m} \log (m+\alpha) \neq 0
$$

for $(\underline{k}, \underline{l}) \neq(\underline{0}, \underline{0})$. Hence, in view of Lemmas 2 and 1 , we obtain by $(2.1)$

$$
\lim _{N \rightarrow \infty} g_{N, \alpha}(\underline{k}, \underline{l})=0
$$

for $(\underline{k}, \underline{l}) \neq(\underline{0}, \underline{0})$. This together with $(2.2)$ shows that

$$
\lim _{N \rightarrow \infty} g_{N, \alpha}(\underline{k}, \underline{l})= \begin{cases}1, & \text { if }(\underline{k}, \underline{l})=(\underline{0}, \underline{0}), \\ 0, & \text { if }(\underline{k}, \underline{l}) \neq(\underline{0}, \underline{0}) .\end{cases}
$$

Since the right-hand side of the latter equality is the Fourier transform of the Haar measure $m_{H}$, a continuity theorem for probability measures on compact groups proves the lemma.

Lemma 3 implies the weak convergence for probability measures defined by means of absolutely convergent Dirichlet series. We recall that $D=\{s \in$ $\left.\mathbb{C}: \frac{1}{2}<\sigma<1\right\}$. Denote by $H(D)$ the space of analytic functions on $D$ endowed with the topology of uniform convergence on compacta, and $H^{2}(D)=$ $H(D) \times H(D)$.

Let $\theta>\frac{1}{2}$ be a fixed number, and, for $m, n \in \mathbb{N}$,

$$
v_{n}(m)=\exp \left\{-\left(\frac{m}{n}\right)^{\theta}\right\},
$$

and, for $m \in \mathbb{N}_{0}, n \in \mathbb{N}$,

$$
v_{n}(m, \alpha)=\exp \left\{-\left(\frac{m+\alpha}{n+\alpha}\right)^{\theta}\right\} .
$$

Define the series

$$
\zeta_{n}(s ; \mathfrak{a})=\sum_{m=1}^{\infty} \frac{a_{m} v_{n}(m)}{m^{s}}, \quad \zeta_{n}(s, \alpha ; \mathfrak{b})=\sum_{m=0}^{\infty} \frac{b_{m} v_{n}(m, \alpha)}{(m+\alpha)^{s}} .
$$

The latter series are absolutely convergent for $\sigma>\frac{1}{2}$ [9]. Moreover, we set

$$
\zeta_{n}\left(s, \omega_{1} ; \mathfrak{a}\right)=\sum_{m=1}^{\infty} \frac{a_{m} \omega_{1}(m) v_{n}(m)}{m^{s}}
$$


and

$$
\zeta_{n}\left(s, \alpha, \omega_{2} ; \mathfrak{b}\right)=\sum_{m=0}^{\infty} \frac{b_{m} \omega_{2}(m) v_{n}(m, \alpha)}{(m+\alpha)^{s}},
$$

the series again being absolutely convergent for $\sigma>\frac{1}{2}$. For brevity, we put

$$
\begin{aligned}
& \underline{\zeta}_{n}(s, \alpha ; \mathfrak{a}, \mathfrak{b})=\left(\zeta_{n}(s ; \mathfrak{a}), \zeta_{n}(s, \alpha ; \mathfrak{b})\right), \\
& \underline{\zeta}_{n}(s, \alpha, \omega ; \mathfrak{a}, \mathfrak{b})=\left(\zeta_{n}\left(s, \omega_{1} ; \mathfrak{a}\right), \zeta_{n}\left(s, \alpha, \omega_{2} ; \mathfrak{b}\right)\right) .
\end{aligned}
$$

Define the function $u_{n, \alpha}: \Omega \rightarrow H^{2}(D)$ by the formula

$$
u_{n, \alpha}(\omega)=\underline{\zeta}_{n}(s, \alpha, \omega ; \mathfrak{a}, \mathfrak{b}) .
$$

Since the series (2.3) and (2.4) are absolutely convergent for $\sigma>\frac{1}{2}$, the function $u_{n, \alpha}$ is continuous, hence $\left(\mathcal{B}(\Omega), \mathcal{B}\left(H^{2}(D)\right)\right)$ - measurable. Therefore, the measure $m_{H}$ induces on $\left(H^{2}(D), \mathcal{B}\left(H^{2}(D)\right)\right)$ the unique probability measure $m_{H} u_{n, \alpha}^{-1}$ defined, for $A \in \mathcal{B}\left(H^{2}(D)\right)$ by

$$
m_{H} u_{n, \alpha}^{-1}(A)=m_{H}\left(u_{n, \alpha}^{-1} A\right) .
$$

Let, for $A \in \mathcal{B}\left(H^{2}(D)\right)$,

$$
P_{N, n, \alpha}(A)=\frac{1}{N} \#\left\{1 \leq k \leq N: \underline{\zeta}_{n}\left(s+i \gamma_{k} h, \alpha ; \mathfrak{a}, \mathfrak{b}\right) \in A\right\} .
$$

Then we have the following statement.

Lemma 4. Suppose that $\alpha$ is a transcendental number. Then $P_{N, n, \alpha}$ converges weakly to $\hat{P}_{n, \alpha} \stackrel{\text { def }}{=} m_{H} u_{n, \alpha}^{-1}$ as $N \rightarrow \infty$.

Proof. By the definition of $u_{n, \alpha}$,

$$
u_{n, \alpha}\left(\left(p^{-i \gamma_{k} h}: p \in \mathbb{P}\right),\left((m+\alpha)^{-i \gamma_{k} h}: m \in \mathbb{N}_{0}\right)\right)=\underline{\zeta}_{n}\left(s+i \gamma_{k} h, \alpha ; \mathfrak{a}, \mathfrak{b}\right) .
$$

Therefore, for every $A \in \mathcal{B}\left(H^{2}(D)\right)$,

$$
\begin{aligned}
P_{N, n, \alpha}(A)= & \frac{1}{N} \#\{1 \leq k \leq N: \\
& \left.\left(\left(p^{-i \gamma_{k} h}: p \in \mathbb{P}\right),\left((m+\alpha)^{-i \gamma_{k} h}: m \in \mathbb{N}_{0}\right)\right) \in u_{n, \alpha}^{-1} A\right\},
\end{aligned}
$$

i. e., $P_{N, n, \alpha}=Q_{N, \alpha} u_{n, \alpha}^{-1}$, where $Q_{N, \alpha}$ is from Lemma 3 . Thus, the assertion of the lemma is a consequence of Lemma 3, continuity of $u_{n, \alpha}$ and Theorem 5.1 of $[1]$.

\section{$3 \quad$ Mean square estimates}

To pass from $\underline{\zeta}_{n}(s, \alpha ; \mathfrak{a}, \mathfrak{b})$ to $\underline{\zeta}(s, \alpha, \mathfrak{a}, \mathfrak{b})=(\zeta(s ; \mathfrak{a}), \zeta(s, \alpha ; \mathfrak{b}))$, we need a certain approximation result for $\underline{\zeta}(s, \alpha ; \mathfrak{a}, \mathfrak{b})$ by $\zeta_{n}(s, \alpha ; \mathfrak{a}, \mathfrak{b})$. For this aim, some mean 
square estimates are needed. In this step, the estimate (1.4) plays an important role. Equalities (1.1) and (1.2) imply for fixed $\sigma, \frac{1}{2}<\sigma<1$, the estimates

$$
\int_{0}^{T}|\zeta(\sigma+i t ; \mathfrak{a})|^{2} \mathrm{~d} t \ll_{\sigma, \mathfrak{a}} T \text { and } \int_{0}^{T}|\zeta(\sigma+i t, \alpha ; \mathfrak{b})|^{2} \mathrm{~d} t \ll_{\sigma, \alpha, \mathfrak{b}} T \text {. }
$$

Hence, for $\tau \in \mathbb{R}$,

$$
\begin{aligned}
& \int_{0}^{T}|\zeta(\sigma+i t+i \tau ; \mathfrak{a})|^{2} \mathrm{~d} t \ll_{\sigma, \mathfrak{a}} T(1+|\tau|), \\
& \int_{0}^{T}|\zeta(\sigma+i t+i \tau, \alpha ; \mathfrak{b})|^{2} \mathrm{~d} t \ll_{\sigma, \alpha, \mathfrak{b}} T(1+|\tau|) .
\end{aligned}
$$

The above mean square estimates are of continuous type. The following Gallagher lemma connects discrete and continuous mean square estimates for certain functions.

Lemma 5. Suppose that $T_{0}, T \geq \delta>0$ are real numbers, and $\mathfrak{T} \neq \varnothing$ is a finite set in the interval $\left[T_{0}+\frac{\delta}{2}, T_{0}+T-\frac{\delta}{2}\right]$. Define

$$
N_{\delta}(x)=\sum_{t \in \mathfrak{T},|t-x|<\delta} 1
$$

Let $S(x)$ be a complex-valued continuous function on $\left[T_{0}, T_{0}+T\right]$ having a continuous derivative on $\left(T_{0}, T_{0}+T\right)$. Then

$\sum_{t \in \mathfrak{T}} N_{\delta}^{-1}(t)|S(t)|^{2} \leq \frac{1}{\delta} \int_{T_{0}}^{T_{0}+T}|S(x)|^{2} d x+\left(\int_{T_{0}}^{T_{0}+T}|S(x)|^{2} d x \int_{T_{0}}^{T_{0}+T}\left|S^{\prime}(x)\right|^{2} d x\right)^{\frac{1}{2}}$

Proof of the lemma is given in [32], Lemma 1.4.

The asymptotics of $\gamma_{k}$ is given in

Lemma 6. For $k \rightarrow \infty, \gamma_{k} \sim 2 \pi k / \log k$.

Proof of the lemma can be found in [35].

Now, we are in position to obtain discrete mean square estimates for the functions $\zeta(s, \mathfrak{a})$ and $\zeta(s, \alpha ; \mathfrak{b})$.

Lemma 7. Suppose that (1.4) is true. Then, for fixed $\sigma, \frac{1}{2}<\sigma<1$, and $\tau \in \mathbb{R}$,

$$
\begin{aligned}
& \sum_{k=1}^{N} \mid \zeta\left(\sigma+i \gamma_{k} h+i \tau ; \mathfrak{a} \mid \ll_{\sigma, \mathfrak{a}, h} N(1+|\tau|),\right. \\
& \sum_{k=1}^{N} \mid \zeta\left(\sigma+i \gamma_{k} h+i \tau, \alpha ; \mathfrak{b} \mid \ll_{\sigma, \alpha, \mathfrak{b}, h} N(1+|\tau|) .\right.
\end{aligned}
$$


Proof. In view of Lemma $6, \gamma_{k} \leq c_{1} k / \log k$ with some $c_{1}>0$ for all $k \geq 2$. We apply Lemma 5 with $\delta=\operatorname{ch}\left(\log \frac{\log N}{c_{1} N}\right)^{-1}, T_{0}=\gamma_{1} h-\frac{\delta}{2}, T=\gamma_{N} h-T_{0}+\frac{\delta}{2}$ and $\mathfrak{T}=\left\{\gamma_{1} h, \ldots, \gamma_{N} h\right\}$. Then we have by (1.4)

$$
\sum_{l=1}^{N} N_{\delta}\left(\gamma_{l} h\right)=\sum_{l=1}^{N} \sum_{\substack{\gamma_{k} \leq \frac{c_{1} N}{\log N} \\\left|\gamma_{l}-\gamma_{k}\right|<\frac{\delta}{h}}} 1=\sum_{\substack{\gamma_{l}, \gamma_{k} \leq \frac{c_{1} N}{\log N} \\\left|\gamma_{l}-\gamma_{k}\right|<\frac{\delta}{h}}} 1 \ll N .
$$

By the Cauchy integral formula,

$$
\zeta^{\prime}(\sigma+i t+i \tau ; \mathfrak{a})=\frac{1}{2 \pi i} \int_{L} \frac{\zeta(z+i t+i \tau ; \mathfrak{a})}{(z-\sigma)^{2}} \mathrm{~d} z,
$$

where $L$ is the circle with a center $\sigma$ lying in $D$. Hence,

$$
\begin{aligned}
\mid \zeta^{\prime}(\sigma & +i t+i \tau ; \mathfrak{a})\left.\right|^{2} \ll\left|\int_{L} \frac{\zeta^{\prime}(z+i t+i \tau ; \mathfrak{a})}{(z-\sigma)^{2}} \mathrm{~d} z\right|^{2} \ll \int_{L} \frac{|\mathrm{d} z|}{|z-\sigma|^{4}} \\
& \times \int_{L}|\zeta(z+i t+i \tau ; \mathfrak{a})|^{2}|\mathrm{~d} z| \ll \sigma \int_{L}|\zeta(z+i t+i \tau ; \mathfrak{a})|^{2}|\mathrm{~d} z| .
\end{aligned}
$$

Therefore, in view of (3.2),

$$
\begin{aligned}
\int_{0}^{T}\left|\zeta^{\prime}(\sigma+i t+i \tau ; \mathfrak{a})\right|^{2} \mathrm{~d} t & \ll \int_{L}|\mathrm{~d} z| \int_{0}^{T}|\zeta(\Re z+i \Im z+i t+i \tau ; \mathfrak{a})|^{2} \mathrm{~d} t \\
& \ll_{\sigma, \mathfrak{a}} T(1+|\tau|) .
\end{aligned}
$$

Now, this (3.1), (3.3) and Lemma 5 yield, for sufficiently large $N$,

$$
\begin{aligned}
& \sum_{k=1}^{N}\left|\zeta\left(\sigma+i \gamma_{k} h+i \tau ; \mathfrak{a}\right)\right|=\sum_{k=1}^{N} \sqrt{N_{\delta}\left(\gamma_{k} h\right) N_{\delta}^{-1}\left(\gamma_{k} h\right)}\left|\zeta\left(\sigma+i \gamma_{k} h+i \tau ; \mathfrak{a}\right)\right| \\
& \ll\left(\sum_{k=1}^{N} N_{\delta}\left(\gamma_{k} h\right) \sum_{k=1}^{N} N_{\delta}^{-1}\left(\gamma_{k} h\right)\left|\zeta\left(\sigma+i \gamma_{k} h+i \tau ; \mathfrak{a}\right)\right|^{2}\right)^{\frac{1}{2}} \\
& \ll_{\sigma} \sqrt{N}\left(\frac{1}{\delta} \int_{0}^{2 \gamma_{N} h}|\zeta(\sigma+i t+i \tau ; \mathfrak{a})|^{2} \mathrm{~d} t+\left(\int_{0}^{2 \gamma_{N} h}|\zeta(\sigma+i t+i \tau ; \mathfrak{a})|^{2} \mathrm{~d} t\right.\right. \\
& \left.\left.\times \int_{0}^{2 \gamma_{N} h}\left|\zeta^{\prime}(\sigma+i t+i \tau ; \mathfrak{a})\right|^{2} \mathrm{~d} t\right)^{\frac{1}{2}}\right)^{\frac{1}{2}} \ll_{\sigma, \mathfrak{b}, h} N(1+|\tau|) .
\end{aligned}
$$

The bound for the function $\zeta(s, \alpha ; \mathfrak{b})$ is obtained similarly.

\section{Approximation results}

In this section, we will approximate $\underline{\zeta}\left(s+i \gamma_{k} h, \alpha ; \mathfrak{a}, \mathfrak{b}\right)$ by $\underline{\zeta}_{n}\left(s+i \gamma_{k} h, \alpha ; \mathfrak{a}, \mathfrak{b}\right)$ in the mean. For this, we recall the metric in the space $H^{2}(D)$. For $g_{1}, g_{2} \in H(D)$, define

$$
\rho\left(g_{1}, g_{2}\right)=\sum_{l=1}^{\infty} 2^{-l} \frac{\sup _{s \in K_{l}}\left|g_{1}(s)-g_{2}(s)\right|}{1+\sup _{s \in K_{l}}\left|g_{1}(s)-g_{2}(s)\right|},
$$


where $\left\{K_{l}: l \in \mathbb{N}\right\}$ is a sequence of compact subsets of the strip $D$ such that $D=\bigcup_{l=1}^{\infty} K_{l}, K_{l} \subset K_{l+1}$ for $l \in \mathbb{N}$, and if $K \subset D$ is a compact set, then $K \subset K_{l}$ for some $l \in \mathbb{N}$. Then $\rho$ is a metric in $H(D)$ inducing its topology of uniform convergence on compacta. For $\underline{g}_{1}=\left(g_{11}, g_{12}\right), \underline{g}_{2}=\left(g_{21}, g_{22}\right) \in H^{2}(D)$, we set

$$
\underline{\rho}\left(\underline{g}_{11}, \underline{g}_{21}\right)=\max _{1 \leq j \leq 2} \rho\left(g_{1 j}, g_{2 j}\right) \text {. }
$$

Then $\underline{\rho}$ is a metric in $H^{2}(D)$ inducing the product topology.

Lemma 8. Suppose that (1.4) is true. Then

$$
\lim _{n \rightarrow \infty} \limsup _{N \rightarrow \infty} \frac{1}{N} \sum_{k=1}^{N} \underline{\rho}\left(\underline{\zeta}\left(s+i \gamma_{k} h, \alpha ; \mathfrak{a}, \mathfrak{b}\right), \underline{\zeta}_{n}\left(s+i \gamma_{k} h, \alpha ; \mathfrak{a}, \mathfrak{b}\right)\right)=0 .
$$

Proof. By the definition of the metric $\underline{\rho}$, it suffices to prove that

$$
\begin{aligned}
& \lim _{n \rightarrow \infty} \limsup _{N \rightarrow \infty} \frac{1}{N} \sum_{k=1}^{N} \rho\left(\zeta\left(s+i \gamma_{k} h ; \mathfrak{a}\right), \zeta_{n}\left(s+i \gamma_{k} h ; \mathfrak{a}\right)\right)=0, \\
& \lim _{n \rightarrow \infty} \limsup _{N \rightarrow \infty} \frac{1}{N} \sum_{k=1}^{N} \rho\left(\zeta\left(s+i \gamma_{k} h, \alpha ; \mathfrak{b}\right), \zeta_{n}\left(s+i \gamma_{k} h, \alpha ; \mathfrak{b}\right)\right)=0 .
\end{aligned}
$$

Let

$$
l_{n}(s)=\frac{s}{\theta} \Gamma\left(\frac{s}{\theta}\right) n^{s},
$$

where $\theta$ comes from the definition of $v_{n}(m)$, and $\Gamma(s)$ denotes the Euler gammafunction. Then it is known that

$$
\zeta_{n}(s ; \mathfrak{a})=\frac{1}{2 \pi i} \int_{\theta-i \infty}^{\theta+i \infty} \zeta(s+z ; \mathfrak{a}) l_{n}(z) \frac{\mathrm{d} z}{z} .
$$

Denote by $a$ the residue of the function $\zeta(s ; \mathfrak{a})$ at the point $s=1$. Let $\hat{\theta}>0$. Then, by (4.3),

$$
\zeta_{n}(s ; \mathfrak{a})-\zeta(s ; \mathfrak{a})=\frac{1}{2 \pi i} \int_{-\hat{\theta}-i \infty}^{-\hat{\theta}+i \infty} \zeta(s+z ; \mathfrak{a}) l_{n}(z) \frac{\mathrm{d} z}{z}+\frac{a l_{n}(1-s)}{1-s} .
$$

Suppose that $K$ is a fixed compact set of the strip $D$, and take $\varepsilon>0$ such that $\frac{1}{2}+2 \varepsilon \leq \sigma \leq 1-\varepsilon$ for any point $s=\sigma+i v \in K$. Now, let

$$
\hat{\theta}=\sigma-\varepsilon-\frac{1}{2} \text { and } \theta=\frac{1}{2}+\varepsilon .
$$

Then (4.4), implies, for $s \in K$, the inequality

$$
\begin{aligned}
\left|\zeta\left(s+i \gamma_{k} h ; \mathfrak{a}\right)-\zeta_{n}\left(s+i \gamma_{k} h ; \mathfrak{a}\right)\right| \leq & \frac{1}{2 \pi} \int_{-\infty}^{\infty}\left|\zeta\left(s+i \gamma_{k} h-\hat{\theta}+i t\right)\right| \frac{l_{n}(-\hat{\theta}+i t)}{|-\hat{\theta}+i t|} \mathrm{d} t \\
& +\frac{|a| l_{n}\left(1-s-i \gamma_{k} h\right) \mid}{\left|1-s-i \gamma_{k} h\right|} .
\end{aligned}
$$


In the latter integral, take $t$ in place $t+v$. This gives

$$
\begin{aligned}
\mid \zeta(s+ & \left.i \gamma_{k} h ; \mathfrak{a}\right)-\zeta_{n}\left(s+i \gamma_{k} h ; \mathfrak{a}\right)\left|\leq \frac{1}{2 \pi} \int_{-\infty}^{\infty}\right| \zeta\left(\frac{1}{2}+\varepsilon+i\left(t+\gamma_{k} h\right) ; \mathfrak{a}\right) \mid \\
& \times \frac{\left|l_{n}\left(\frac{1}{2}+\varepsilon-s+i t\right)\right|}{\left|\frac{1}{2}+\varepsilon-s+i t\right|} \mathrm{d} t+\frac{|a| l_{n}\left(1-s-i \gamma_{k} h\right) \mid}{\left|1-s-i \gamma_{k} h\right|} .
\end{aligned}
$$

This leads to

$$
\frac{1}{N} \sum_{k=1}^{N} \sup _{s \in K}\left|\zeta\left(s+i \gamma_{k} ; \mathfrak{a}\right)-\zeta_{n}\left(s+i \gamma_{k} h ; \mathfrak{a}\right)\right| \leq S_{1}+S_{2},
$$

where

$$
\begin{aligned}
& S_{1}=\frac{1}{2 \pi N} \int_{-\infty}^{\infty}\left(\sum_{k=1}^{N}\left|\zeta\left(\frac{1}{2}+\varepsilon+i\left(t+\gamma_{k} h\right) ; \mathfrak{a}\right)\right| \sup _{s \in K} \frac{\left|l_{n}\left(\frac{1}{2}+\varepsilon-s+i t\right)\right|}{\left|\frac{1}{2}+\varepsilon-s+i t\right|}\right) \mathrm{d} t, \\
& S_{2}=\frac{|a|}{N} \sum_{k=1}^{N} \sup _{s \in K} \frac{\left|l_{n}\left(1-s-i \gamma_{k} h\right)\right|}{\left|1-s-i \gamma_{k} h\right|} .
\end{aligned}
$$

For the function $\Gamma(\sigma+i t)$, the estimate

$$
\Gamma(\sigma+i t) \ll \exp \{-c|t|\}, c>0
$$

uniform in $\sigma_{1} \leq \sigma \leq \sigma_{2}$, is known. Therefore, the definition of the function $l_{n}(s)$ implies the bound, for $s \in K$,

$$
\frac{l_{n}\left(\frac{1}{2}+\varepsilon-s+i t\right)}{\frac{1}{2}+\varepsilon-s+i t} \ll n^{-\varepsilon} \exp \left\{-\frac{c|t-v|}{\theta}\right\} \ll_{K} n^{-\varepsilon} \exp \{-c|t|\} .
$$

By similar arguments, we find that

$$
\frac{l_{n}\left(1-s-i \gamma_{k} h\right)}{1-s-i \gamma_{k} h} \ll_{K, h} n^{1-\sigma} \exp \left\{-c \gamma_{k} h\right\}
$$

From (4.6) and Lemma 7, it follows that

$$
S_{1} \ll_{K, \mathfrak{a}, h} n^{-\varepsilon} \int_{-\infty}^{\infty}(1+|t|) \exp \{-c|t|\} \mathrm{d} t \ll_{K, \mathfrak{a}, h} n^{-\varepsilon},
$$

while (4.7) shows that

$$
S_{2} \ll_{K, \mathfrak{a}, n} n^{\frac{1}{2}-2 \varepsilon} \frac{\log N}{N} .
$$

Therefore, in view of (4.5), we obtain that

$$
\lim _{n \rightarrow \infty} \limsup _{N \rightarrow \infty} \frac{1}{N} \sum_{k=1}^{N} \sup _{s \in K}\left|\zeta\left(s+i \gamma_{k} h ; \mathfrak{a}\right)-\zeta_{n}\left(s+i \gamma_{k} h ; \mathfrak{a}\right)\right|=0,
$$

and this and the definition of the metric $\rho$ imply (4.1). 
The equality (4.2) is proved similarly by using the representation

$$
\zeta_{n}(s, \alpha ; \mathfrak{b})=\frac{1}{2 \pi i} \int_{-\theta+i \infty}^{\theta+i \infty} \zeta(s+z, \alpha ; \mathfrak{b}) \frac{l_{n}(z, \alpha)}{z} d z
$$

where

$$
l_{n}(s, \alpha)=\frac{s}{\theta} \Gamma\left(\frac{s}{\theta}\right)(n+\alpha)^{s},
$$

as well as the second bound of Lemma 7 .

\section{$5 \quad$ A limit theorem}

In this section, we will prove a limit theorem for $\zeta(s, \alpha ; \mathfrak{a}, \mathfrak{b})$ in the space $H^{2}(D)$. For the statement of that theorem, a certain $H^{2}(D)$ - valued random element is used. On the probability space $\left(\Omega, \mathcal{B}(\Omega), m_{H}\right)$, define the $H^{2}(D)$ - valued random element

$$
\underline{\zeta}(s, \omega, \alpha ; \mathfrak{a}, \mathfrak{b})=\left(\sum_{m=1}^{\infty} \frac{a_{m} \omega_{1}(m)}{m^{s}}, \sum_{m=0}^{\infty} \frac{b_{m} \omega_{2}(m)}{(m+\alpha)^{s}}\right) .
$$

We observe that the latter series both are almost surely uniformly convergent on compact subsets of the strip $D$. Denote by $P_{\underline{\zeta}, \alpha}$ the distribution of the random element $\underline{\zeta}(s, \omega, \alpha ; \mathfrak{a}, \mathfrak{b})$, i. e.,

$$
P_{\underline{\zeta}, \alpha}(A)=m_{H}\{\omega \in \Omega: \underline{\zeta}(s, \omega, \alpha ; \mathfrak{a}, \mathfrak{b}) \in A\}, A \in \mathcal{B}\left(H^{2}(D)\right) .
$$

Moreover, for $A \in \mathcal{B}\left(H^{2}(D)\right)$,

$$
P_{N, \alpha}(A)=\frac{1}{N} \#\left\{1 \leq k \leq N: \underline{\zeta}\left(s+i \gamma_{k} h, \alpha ; \mathfrak{a}, \mathfrak{b}\right) \in A\right\} .
$$

Theorem 3. Suppose that the sequence $\mathfrak{a}$ is multiplicative, the parameter $\alpha$ is transcendental, and the bound (1.4) is true. Then $P_{N, \alpha}$ converges weakly to $P_{\underline{\zeta}, \alpha}$ as $N \rightarrow \infty$.

Proof. We return to Lemma 4 and its limit measure $\hat{P}_{n, \alpha}$. Let $\theta_{N}$ be a random variable defined on a certain probability space with the measure $\mu$ and having the distribution

$$
\mu\left\{\theta_{N}=\gamma_{k} h\right\}=\frac{1}{N}, k=1, \ldots, N .
$$

Define the $H^{2}(D)$ - valued random element

$$
X_{N, n, \alpha}=X_{N, n, \alpha}(s)=\underline{\zeta}_{n}\left(s+i \theta_{N}, \alpha ; \mathfrak{a}, \mathfrak{b}\right) .
$$

Then, denoting by $\hat{X}_{n, \alpha}$ the $H^{2}(D)$-valued random element with the distribution $\hat{P}_{n, \alpha}$, we rewrite the assertion of Lemma 4 in the form 


$$
X_{N, n, \alpha} \underset{N \rightarrow \infty}{\stackrel{\mathcal{D}}{\longrightarrow}} \hat{X}_{n, \alpha}
$$

In [9], it is proved that the sequence of probability measures $\left\{\hat{P}_{n, \alpha}: n \in \mathbb{N}\right\}$ is tight, i.e., for every $\varepsilon>0$, there exists a compact set $K=K(\varepsilon) \subset H^{2}(D)$ such that

$$
\hat{P}_{n, \alpha}(K)>1-\varepsilon
$$

for all $n \in \mathbb{N}$. By the Prokhorov theorem [1], Theorem 6.1, every tight family of probability measures is relatively compact. Thus, every subsequence of $\left\{\hat{P}_{n, \alpha}\right\}$ contains a subsequence $\left\{\hat{P}_{n_{r}, \alpha}\right\}$ such that $\hat{P}_{n_{r}, \alpha}$ converges weakly to a certain probability measure $P_{\alpha}$ on $\left(H^{2}(D), B\left(H^{2}(D)\right)\right)$ as $r \rightarrow \infty$. This also can be writen in the form

$$
\hat{X}_{n_{r}, \alpha} \underset{r \rightarrow \infty}{\stackrel{\mathcal{D}}{\longrightarrow}} P_{\alpha}
$$

Using the random variable $\theta_{N}$, define one more $H^{2}(D)$-valued random element

$$
X_{N, \alpha}=X_{N, \alpha}(s)=\underline{\zeta}\left(s+i \theta_{N}, \alpha ; \mathfrak{a}, \mathfrak{b}\right) .
$$

Then Lemma 8 implies, for every $\varepsilon>0$,

$$
\begin{aligned}
\lim _{n \rightarrow \infty} \limsup _{N \rightarrow \infty} \mu\left\{\underline{\rho}\left(X_{N, \alpha}, X_{N, n, \alpha}\right) \geq \varepsilon\right\} \\
=\lim _{n \rightarrow \infty} \limsup _{N \rightarrow \infty} \frac{1}{N} \#\{1 \leq k \leq N: \\
\left.\left.\quad \underline{\rho} \underline{\zeta}\left(s+i \gamma_{k} h, \alpha ; \mathfrak{a}, \mathfrak{b}\right), \underline{\zeta}_{n}\left(s+i \gamma_{k} h, \alpha ; \mathfrak{a}, \mathfrak{b}\right)\right) \geq \varepsilon\right\} \\
\leq \lim _{n \rightarrow \infty} \limsup _{N \rightarrow \infty} \frac{1}{N \varepsilon} \sum_{k=1}^{N} \underline{\rho}\left(\underline{\zeta}\left(s+i \gamma_{k} h, \alpha ; \mathfrak{a}, \mathfrak{b}\right), \underline{\zeta}_{n}\left(s+i \gamma_{k} h, \alpha ; \mathfrak{a}, \mathfrak{b}\right)\right)=0 .
\end{aligned}
$$

Now, this equality, the relations (5.1) and (5.2), and Theorem 4.2 of [1] show that

$$
X_{N, \alpha} \underset{N \rightarrow \infty}{\stackrel{\mathcal{D}}{\longrightarrow}} P_{\alpha},
$$

or $P_{N, \alpha}$ converges weakly to $P_{\alpha}$ as $N \rightarrow \infty$. Moreover, the latter relation shows that the measure $P_{\alpha}$ is independent of the subsequence $\left\{\hat{P}_{n_{r}}, \alpha\right\}$. This remark gives the relation

$$
\hat{X}_{n, \alpha} \underset{n \rightarrow \infty}{\stackrel{\mathcal{D}}{\longrightarrow}} P_{\alpha},
$$

or $\hat{P}_{n, \alpha}$ converges weakly to $P_{\alpha}$ as $n \rightarrow \infty$. Thus, we obtained that $P_{N, \alpha}$ converges weakly to the limit measure $P_{\alpha}$ of $\hat{P}_{n, \alpha}$. In [15], it was shown that $P_{\alpha}$ coincides with $P_{\underline{\zeta}, \alpha}$. 


\section{Proof of universality theorems}

Let

$$
S=\{g \in H(D): g(s) \neq 0 \text { or } g(s) \equiv 0\} \times H(D) .
$$

Then it was proved in [9] that the support of the measure $P_{\zeta, \alpha}$ is the set $S$.

Proof of Theorem 1. By the Mergelyan theorem on the approximation analytic functions by polynomials [31], there exist polynomials $p_{1}(s)$ and $p_{2}(s)$ such that

$$
\begin{aligned}
& \sup _{s \in K_{1}}\left|f_{1}(s)-e^{p_{1}(s)}\right|<\frac{\varepsilon}{2}, \\
& \sup _{s \in K_{2}}\left|f_{2}(s)-p_{2}(s)\right|<\frac{\varepsilon}{2} .
\end{aligned}
$$

Define

$$
G_{\varepsilon}=\left\{\left(g_{1}, g_{2}\right) \in H^{2}(D): \sup _{s \in K_{1}}\left|g_{1}(s)-e^{p_{1}(s)}\right|<\frac{\varepsilon}{2}, \sup _{s \in K_{2}}\left|g_{2}(s)-p_{2}(s)\right|<\frac{\varepsilon}{2}\right\} .
$$

Then $G_{\varepsilon}$ is an open neighbourhood of the element $\left(e^{p_{1}(s)}, p_{2}(s)\right) \in S$. Therefore, by properties of the support,

$$
P_{\underline{\zeta}, \alpha}\left(G_{\varepsilon}\right)>0 .
$$

Moreover, by Theorem 3 and the equivalent of weak convergence of probability measure in terms of open sets [1], Theorem 2.1, we have that

$$
\liminf _{N \rightarrow \infty} P_{N, \alpha}\left(G_{n}\right) \geq P_{\underline{\zeta}, \alpha}\left(G_{\varepsilon}\right)>0 .
$$

This, the definitions of $P_{N, \alpha}$ and $G_{\varepsilon}$ together with (6.1) and (6.2) prove the theorem.

Proof of Theorem 2. Define

$$
\hat{G}_{\varepsilon}=\left\{\left(g_{1}, g_{2}\right) \in H^{2}(D): \sup _{1 \leq j \leq 2} \sup _{s \in K_{j}}\left|g_{j}(s)-f_{j}(s)\right|<\varepsilon\right\} .
$$

Then the boundary $\partial \hat{G}_{\varepsilon}$ of $\hat{G}_{\varepsilon}$ lies in the set

$$
\left\{\left(g_{1}, g_{2}\right) \in H^{2}(D): \sup _{1 \leq j \leq 2} \sup _{s \in K_{j}}\left|g_{j}(s)-f_{j}(s)\right|=\varepsilon\right\} .
$$

Therefore, the boundaries $\partial \hat{G}_{\varepsilon_{1}}$ and $\partial \hat{G}_{\varepsilon_{2}}$ with different positive $\varepsilon_{1}$ and $\varepsilon_{2}$ do not intersect. Hence, the set $\hat{G}_{\varepsilon}$ is a continuity set $\left(P_{\underline{\zeta}, \alpha}\left(\partial \hat{G}_{\varepsilon}\right)=0\right)$ of the measure $P_{\zeta, \alpha}$ for all but at most countably many $\varepsilon>0$. Therefore, by Theorem 3 and the equivalent of weak convergence of probability measures in terms of continuity sets [1], Theorem 2.1, we have that

$$
\lim _{n \rightarrow \infty} P_{N, \alpha}\left(\hat{G}_{\varepsilon}\right)=P_{\underline{\zeta}, \alpha}\left(\hat{G}_{\varepsilon}\right)
$$


for all but at most countably many $\varepsilon>0$. The definitions of $G_{\varepsilon}$ and $\hat{G}_{\varepsilon}$, and (6.1) and (6.2) show that $G_{\varepsilon} \subset \hat{G}_{\varepsilon}$. Thus, in view of (6.3),

$$
P_{\underline{\zeta}, \alpha}\left(\hat{G}_{\varepsilon}\right)>0 \text {. }
$$

This, the definitions of $P_{N, \alpha}$ and $\hat{G}_{\varepsilon}$ together with (6.4) give the assertion of the theorem.

\section{References}

[1] P. Billingsley. Convergence of Probability Measures. John Wiley and Sons, New York, 1968.

[2] V. Franckevič, A. Laurinčikas and D. Šiaučiūnas. On approximation of analytic functions by periodic Hurwitz zeta-functions. Math. Modell. Analysis, 24(1):2033, 2019. https://doi.org/10.3846/mma.2019.002.

[3] V. Garbaliauskienè, J. Karaliūnaitè and R. Macaitienè. On discrete value distribution of certain compositions. Math. Modell. Analysis, 24(1):34-42, 2019. https://doi.org/10.3846/mma.2019.003.

[4] R. Garunkštis and A. Laurinčikas. Discrete mean square of the Riemann zetafunction over imaginary parts of its zeros. Periodica Math. Hung., 76(2):217-228, 2018. https://doi.org/10.1007/s10998-017-0228-6.

[5] R. Garunkštis and A. Laurinčikas. The Riemann hypothesis and universality of the Riemann zeta-function. Math. Slovaca, 68(4):741-748, 2018. https://doi.org/10.1515/ms-2017-0141.

[6] R. Garunkštis, A. Laurinčikas and R. Macaitienè. Zeros of the Riemann zeta-function and its universality. Acta Arith., 181(2):127-142, 2017. https://doi.org/10.4064/aa8583-5-2017.

[7] A. Javtokas and A. Laurinčikas. Universality of the periodic Hurwitz zeta-function. Integral Transf. Spec. Functions, 17:711-722, 2006. https://doi.org/10.1080/10652460600856484.

[8] J. Kaczorowski. Some remarks on the universality of periodic $L$-functions. In R. Steuding and J. Steuding(Eds.), New directions in value-distribution theory of zeta and L-functions, pp. 113-120. Shaker Verlag, Aachen, 2009.

[9] R. Kačinskaitè and A. Laurinčikas. The joint distribution of periodic zeta-functions. Studia Sci. Math. Hungarica, 48(2):257-279, 2011. https://doi.org/10.1556/SScMath.48.2011.2.1162.

[10] L. Kuipers and H. Niederreiter. Uniform Distribution of Sequences. John Wiley and Sons, New York, 1974.

[11] A. Laurinčikas. Voronin-type theorem for periodic Hurwitz zeta-functions. $\quad S b . \quad$ Math., $198: 231-262, \quad 2007$. https://doi.org/10.1070/SM2007v198n02ABEH003835.

[12] A. Laurinčikas. Joint universality for periodic Hurwitz zeta-functions. Izv. Math., 72:741-760, 2008. https://doi.org/10.1070/IM2008v072n04ABEH002421.

[13] A. Laurinčikas. Joint universality of zeta-functions with

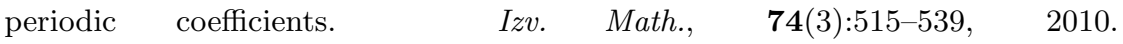
https://doi.org/10.1070/IM2010v074n03ABEH002497. 
[14] A. Laurinčikas. Universality of composite functions of periodic zeta-functions. $\quad S b . \quad$ Math., $203: 1631-1643,2012$. https://doi.org/10.1070/SM2012v203n11ABEH004279.

[15] A. Laurinčikas. The joint discrete universality of periodic zeta-functions. In J. Sander et al.(Ed.), From Arithmetic to Zeta-Functions, Number Theory in Memory of Wolfgang Schwarz, pp. 231-246. Springer, 2016. https://doi.org/10.1007/978-3-319-28203-9_15.

[16] A. Laurinčikas. Universality theorems for zeta-functions with periodic coefficients. Sb. Math. J., $\quad \mathbf{5 7}: 330-339,2016$. https://doi.org/10.1134/S0037446616020154.

[17] A. Laurinčikas. A discrete version of the Mishou theorem. II. Proc. Steklov Inst. Math., 296(1):172-182, 2017. https://doi.org/10.1134/S008154381701014X.

[18] A. Laurinčikas. On discrete universality of the Hurwitz zeta-functions. Results Math., 72(1-2):907-917, 2017. https://doi.org/10.1007/s00025-017-0702-8.

[19] A. Laurinčikas. Joint value distribution theorems for the Riemann and Hurwitz zeta-functions. Moscow Math. J., 18(2):349-366, 2018. https://doi.org/10.17323/1609-4514-2018-18-2-349-366.

[20] A. Laurinčikas. Joint discrete universality for periodic zeta-functions. Quaest. Math., 42(5):687-699, 2019. https://doi.org/10.2989/16073606.2018.1481891.

[21] A. Laurinčikas. Non-trivial zeros of the Riemann zeta-function and joint universality theorems. J. Math. Anal. Appl., 475(1):395-402, 2019. https://doi.org/10.1016/j.jmaa.2019.02.047.

[22] A. Laurinčikas and R. Macaitienè. The discrete universality of the periodic Hurwitz zeta-function. Integral Transf. Spec. Functions, 20:673-686, 2009. https://doi.org/10.1080/10652460902742788.

[23] A. Laurinčikas and R. Macaitienè. Joint approximation of analytic functions by shifts of the Riemann and periodic Hurwitz zeta-functions. Appl. Anal. Discrete Math., 12(2):508-527, 2018. https://doi.org/10.2298/AADM170713016L.

[24] A. Laurinčikas, R. Macaitienè, D. Mochov and D. Šiaučiūnas. Universality of the periodic Hurwitz zeta-function with rational parameter. Siber. Math. J., 59(5):894-900, 2018. https://doi.org/10.1134/S0037446618050130.

[25] A. Laurinčikas and D. Mochov. A discrete universality theorem for the periodic Hurwitz zeta-functions. Chebysh. Sb., 17:148-159, 2016.

[26] A. Laurinčikas and J. Petuškinaitè. Universality of Dirichlet $L$-functions and non-trivial zeros of the Riemann zeta-function. Sb. Math., 210, 2019. https://doi.org/10.1070/SM9194.

[27] A. Laurinčikas and D. Šiaučiūnas. Remarks on the universality of periodic zeta-functions (in Russian). Math. Notes., 80(3-4):532-538, 2006. https://doi.org/10.1007/s11006-006-0171-y.

[28] R. Macaitienė, M.Stoncelis and Šiaučiūnas. A weighted discrete universality theorem for periodic zeta-functions. II. Math. Modell. Analysis, 22(6):750-762, 2017. https://doi.org/10.3846/13926292.2017.1365779.

[29] R. Macaitienè, M.Stoncelis and D. Šiaučiūnas. A weighted universality theorem for periodic zeta-functions. Math. Modell. Analysis, 22(1):95-105, 2017. https://doi.org/10.3846/13926292.2017.1269373. 
[30] R. Macaitienè and D. Šiaučiūnas. Joint universality of Hurwitz zeta-functions and nontrivial zeros of the Riemann zeta-function. Lith. Math. J., 59(1):81-95, 2019. https://doi.org/10.1007/s10986-019-09423-2.

[31] S.N. Mergelyan. Uniform approximations to functions of complex variable. Usp. Mat. Nauk., 7(2):31-122, 1952 (in Russian).

[32] H. L. Montgomery. Topics in Multiplicative Number Theory. Lect. Notes Math., Vol. 227, Springer, Berlin, Heidelberg, New York, 1971. https://doi.org/10.1007/BFb0060851.

[33] H. L. Montgomery. The pair correlation of zeros of the zeta function. In H.G. Diamond(Ed.), Analytic Number Theory, volume 24 of Proc. Sympos. Pure Math., pp. 181-193. Amer. Math. Soc., Providence, RI, 1973. https://doi.org/10.1090/pspum/024/9944.

[34] J. Steuding. The roots of the equation $\zeta(s)=a$ are uniformly distributed modulo one. In A. Laurinčikas et al.(Ed.), Analytic and Probabilistic Methods in Number Theory, pp. 243-249, Vilnius, 2012. TEV.

[35] E. C. Titchmarsh. The Theory of the Riemann zeta-function. 2nd ed. revised by D.R. Heath-Brown, Clarendon Press, Oxford, 1986. 